\title{
Pseudo-Hermitian Operators in a Description of Physical Systems
}

V. Jakubský

We present some basic features of pseudo-hermitian quantum mechanics and illustrate the use of pseudo-hermitian Hamiltonians in a description of physical systems.

Keywords: pseudo-hermitian operators, Klein-Gordon and Proca equation, thermodynamics.

\section{Scalar products and metric operators}

In classical mechanics, the system is described by its coordinates in $\mathrm{N}$-dimensional phase (configuration) space. The unique trajectory is a solution of canonical Hamiltonian or Euler-Lagrange equations and satisfies fixed initial conditions. The observable quantities (energy, angular momentum, ...) of the systems are represented by a function on the phase space.

The description of the system is completely different in quantum theory. We are dealing with a infinite dimensional Hilbert space in this case, which is a vector space complete with respect to the norm induced by the associated scalar product. The quantum mechanical system is represented by a vector in the Hilbert space. The observables are represented by the operator acting on the Hilbert space. By postulate, the measurable quantities are associated with spectra of the operators which are required to be real. For this reason, only self-adjoint operators are considered to be physically admissible.

The evolution equations of classical mechanics are replaced by the Schrödinger equation

$$
i \hbar \frac{\partial}{\partial t} \psi=H \psi
$$

and the initial conditions are replaced by the requirement that $\psi$ lies in the domain of the Hamiltonian. The task of solving the evolution equations is more complicated in quantum mechanics. From the mathematical point of view, we face a second order differential equation of complex valued functions. Additionally, the involved self-adjoint Hamiltonians are mostly unbounded, which requires careful determination of their domains.

The scalar product plays the key role in interpreting the solutions of the Schrödinger equation. The quantity $\left(\psi_{1}, \psi_{2}\right)$ is proportional to the transition amplitude that the system in state $\psi_{1}$ will transform to the state described by the vector $\psi_{2}$.

When the system does not depend on time explicitly, the measured quantities have to be independent with respect to time translations. Particularly, the time evolution of the states $\psi_{i}(t)=U(t) \psi_{i}(0)$ should not violate the transition amplitude. In other words, there should hold

$$
\left(\psi_{1}(0), \psi_{2}(0)\right)=\left(U(t) \psi_{1}(0), U(t) \psi_{2}(0)\right)
$$

This implies that the time evolution operator has to be unitary, i.e. $U^{\dagger}(t) U(t)=1$. The dagger denotes hermitian con- jugation with respect to the scalar product (., .) , which is usually taken to be

$$
\left(\psi_{1}, \psi_{2}\right)=\int \psi_{1}^{*} \psi_{2} \mathrm{~d} q^{N}
$$

where $*$ is complex conjugation. The time evolution operators form a one-parameter group, $U(0)=1$, $U\left(t_{1}, t_{2}\right)=U\left(t_{1}\right) U\left(t_{2}\right)$.

The Hamiltonian of the system plays the role of the generator of time translations, we have $U(t)=\exp (-i H t)$. The relation is consistent with Stone's theorem which establishes a correspondence between self-adjoint operators and one-parameter groups of unitary operators.

A few years ago, we witnessed a boom in the study of $\mathcal{P} \mathcal{T}$-symmetric Hamiltonians [1-21]. These operators are specific by their $\mathcal{P} \mathcal{T}$-symmetry, i.e. $[\mathcal{P} \mathcal{T}, H]=0$, where $\mathcal{P}$ is space reflection and $\mathcal{T}$ is time reversion. These can be covered by a broad class of pseudo-hermitian operators which satisfy the following operator equation

$$
H^{\dagger}=\eta H \eta^{-1}
$$

The operator $\eta$ is required to be hermitian, invertible and bounded. The standard self-adjointness is a special case of the preceeding relation as long as $\eta=1$.

It was observed that these operators possess a real spectrum in many cases. The natural question emerged whether it would be possible to replace the standard requirement of self-adjointness by the less restrictive requirement of pseudo-hermiticity.

The answer showed to be affirmative as long as we are dealing with operators having purely real spectra. At first sight, we encounter a serious problem that the time evolution generated by these Hamiltonians is non-unitary with respect to the scalar product (1). This problem has been fixed by the theorem which states [14]: for any pseudo-hermitian operator with a purely real spectrum there exists a positive-definite operator $\Theta$ with respect to which the Hamiltonian is pseudo-hermitian, i.e.

$$
H^{\dagger} \Theta=\Theta H, \quad \Theta>0
$$

The consistent unitary evolution generated by $H$ is recovered by redefining of the scalar product. Instead of (1), we fix it in the following form

$$
\left(\psi_{1}, \psi_{2}\right)_{\Theta}=\int \psi_{1}^{*} \Theta \psi_{2} \mathrm{~d} q^{N}
$$


The Hamiltonian $H$ is self-adjoint with respect to the new scalar product and may consequently serve as a generator of time translations.

So, we encounter a twofold problem in pseudo-hermitian quantum mechanics.

First, one has to check the spectrum of the operator. When it consists of real eigenvalues only, the existence of positive-definite metric operator $\Theta$ is guaranteed and its explicit construction follows. The construction is non-trivial in most cases and contains an additional subtlety: the resulting metric operator is non-unique. For given Hamiltonian $H$, there exist rather a class of admissible metric operators and associated scalar products.

The mathematical framework does not provide any restriction to make the result of the construction unique. To weaken the ambiguity, one employs additional, physically motivated requirements on the scalar product - metric operator.

In the following section, we will provide an example of pseudo-hermitian systems which appear in relativistic quantum mechanics. It illustrates the construction of a proper positive-definite scalar-product and discusses consequent restriction of its ambiguity by the additional physical requirement of relativistic invariance.

\section{Pseudo-hermitian Hamiltonians in relativistic quantum mechanics}

Attempts to marry the special theory of relativity (STR) and quantum mechanics gave rise to the well known equations of relativistic quantum mechanics. The theory built on the Dirac equation provides a consistent description of relativistic spin one-half particles, at least in the presence of weak external fields.

The theory based on Klein-Gordon and Proca equations representing evolution equations for spin zero and spin one particles has met serious problems with probabilistic interpretation of their solutions. The associated scalar product showed to be indefinite. The problem is closely related to the fact that the equations are of the second order in the time derivative.

Nowadays, the genuine fusion of STR and quantum theory is represented by quantum field theory. However, relativistic quantum mechanics may serve as a powerful tool in describing compound particles in the weak external fields or may provide relativistic corrections to the non-relativistic systems.

Recently, relativistic integer-spin systems have been reconsidered within the framework of pseudo-hermitian quantum mechanics. The positive scalar product for both cases has been constructed $[15,16]$. The dynamics of the free spin-less particle is governed by the well-kown Klein-Gordon equation

$$
\left(\partial_{t}^{2}-p^{2}-m^{2}\right) \psi=0 .
$$

The first derivative of the wave function in time can be understood as a new physical degree of freedom. In this context, we introduce the following denotation

$$
i \partial_{t} \psi=\phi \text {. }
$$

Together with this relation, eq. (3) turns to a couple of mutually intertwined differential equations of functions $\phi$ and $\psi$. This system can be rewritten in terms of two-compo- nental formalism into the compact form, which resembles the Schrödinger equation

$i \partial_{t} \Psi=i \partial_{t}\left(\begin{array}{l}\psi \\ \phi\end{array}\right)=\left(\begin{array}{cc}0 & 1 \\ m^{2}+p^{2} & 0\end{array}\right)\left(\begin{array}{l}\psi \\ \phi\end{array}\right)=H \Psi$.

It was shown a long time ago [22] that the relativistic evolution equations can be unified formally in the framework of $2(2 s+1)$-component formalism, where $s$ denotes spin of the particle. Spin-one half keeps its privileged position even in this framework, as the associated Hamiltonian (Dirac Hamiltonian) is hermitian in contrast to the integer-spin systems.

The operator $H$ ceases to be hermitian. Instead, it satisfies the relation

$$
H^{\dagger}=P H P, \quad P=\left(\begin{array}{ll}
0 & 1 \\
1 & 0
\end{array}\right),
$$

which establishes its pseudo-hermiticity with respect to $P$.

The Hamiltonian has a purely real spectrum which consists of the values $\pm \omega= \pm \sqrt{p^{2}+m^{2}}$. Consequently, the existence of the positive metric operator is guaranteed. Its explicit form in momentum representation reads

$\Theta(p)=\frac{1}{\omega}\left(\begin{array}{cc}\omega\left(\theta_{+}(p)+\theta_{-}(p)\right) & -\theta_{+}(p)+\theta_{-}(p) \\ -\theta_{+}(p)+\theta_{-}(p) & \frac{\theta_{+}(p)+\theta_{-}(p)}{\omega}\end{array}\right)$.

This contains positive but arbitrary functions $\theta_{ \pm}(p)$ which represent the non-uniquness of the scalar product. To restrict the ambiguity, we impose additional requirement of Lorentz invariance of the scalar product

$$
\left(\Psi_{1}^{\prime}, \Theta \Psi_{2}^{\prime}\right)=\left(\Psi_{1}, \Theta \Psi_{2}\right),
$$

which in terms of infinitesimal transformations

$$
\Psi^{\prime}=(1+i \varepsilon M) \Psi, \quad \varepsilon: 0
$$

leads to

$$
M^{\dagger} \Theta=\Theta M .
$$

$M$ is a generator of the Poincare group in appropriate representation. Instead of explicit computation, let us note that the solution of the preceeding relation can be particularly facilitated by the use of Shur's lemma $[15,16]$.

The additional, physically motivated constraint reduces the ambiguity to a single parameter. Thus, we may write the resulting scalar product of two solutions $\psi_{i}$ of the Klein-Gordon equation in the following form

$$
\begin{aligned}
\left(\left(\psi_{1}, \psi_{2}\right)\right) \equiv & \left(\Psi_{1}, \Theta \Psi_{2}\right)=\frac{1}{2 m}\left(\left\langle\psi_{1}, \omega \psi_{2}\right\rangle+\left\langle\dot{\psi}_{1}, \frac{1}{\omega} \dot{\psi}_{2}\right\rangle\right) \\
& +\frac{i \alpha}{m}\left(\left\langle\psi_{1}, \dot{\psi}_{2}\right\rangle-\left\langle\dot{\psi}_{1}, \psi_{2}\right\rangle\right), \alpha \in(-1,1) .
\end{aligned}
$$

The system involving a free particle with spin-one can be treated in a similar manner. The evolution equation

$$
\partial_{\mu} F^{\mu v}+m^{2} A^{v}=0
$$

where $F^{\mu v}=\partial^{\mu} A^{v}-\partial^{v} A^{\mu}$ and $A^{\mu}$ are complex valued functions - components of a four-potential, can be rewritten in multi-component formalism as well. Introducing a 6-componential wave function $\Psi^{T}=(m \vec{A}, i \vec{E})^{T}$ we obtain (we define $F^{0 j}=-E_{j}$ for $\left.j=1,2,3\right)$ 
$i \frac{\partial}{\partial t} \Psi=\left(\begin{array}{cc}0 & -m+\frac{\operatorname{grad} \operatorname{div}}{m} \\ -m+\frac{\nabla^{2}}{m}-\frac{\operatorname{grad} \operatorname{div}}{m} & 0\end{array}\right) \Psi=H \Psi$

which is pseudo-hermitian with respect to $P$. Its spectrum is real, and the existence of the positive metric operator is guaranteed again. After explicit construction of the metric operator, we impose the requirement of relativistic invariance of the scalar product. As a result, we obtain a one-parameter family of the scalar product [16]. Its explicit form on the space of solutions $A_{j}=\left(A_{j}^{0}, \vec{A}_{j}\right)^{T}, j=1,2$ of the Proca equations is

$\left(\left(A_{1}, A_{2}\right)\right) \equiv-\frac{i a}{2 m}\left[\left\langle\vec{A}_{1}, \vec{A}_{2}+\operatorname{grad} A_{2}^{0}\right\rangle-\left\langle\vec{A}_{1}+\operatorname{grad} A_{1}^{0}, \vec{A}_{2}\right\rangle\right]$

$+\left\langle\vec{A}_{1}+\operatorname{grad} A_{1}^{0},\left[\frac{\hat{\omega}}{2 m^{3}} I-\frac{p^{2} \hat{h}^{2}}{2 m^{3} \hat{\omega}}\right]\left(\vec{A}_{2}+\operatorname{grad} A_{2}^{0}\right)\right\rangle$

$+\left\langle\vec{A}_{1},\left[\frac{m}{\hat{\omega}} I+\frac{p^{2} \hat{h}^{2}}{2 m \hat{\omega}}\right] \vec{A}_{2}\right\rangle$,

where $\alpha$ in $(-1,1),\langle.,$.$\rangle denotes standard scalar product and$ $\hat{\omega}=\int \mathrm{d} p \omega|p\rangle\langle p|$ and $\hat{h}$ is the helicity operator.

Formulas (11) and (14) set up the basis for direct computation of observable physical properties of the system of free relativistic particles with spin zero and one.

\section{Metric operator in thermodynamics}

In the preceeding section, we considered a system which involved a single particle described by the Hamiltonian $H$. The scalar products played the crucial role in computing the physical properties of these systems. The situation is different when we are interested in the collective behavior of identical systems. A statistical description of the ensemble of quantum systems comes into play.

Let us assume that the system in equilibrium is described by pseudo-hermitian Hamiltonian with a purely real spectrum. Then the state of the system is described by density matrix $\rho$, which is the solution of the Bloch equation

$$
\frac{\partial \rho}{\partial \beta}=-H \rho
$$

with initial condition $\rho(0)=1$. The parameter $\beta$ equals inverse temperature, i.e. $\beta=1 / T$. The density matrix fixes the probability that we find the system in a given state. It provides only statistical information on the system in this sense.

The formal solution of the Bloch equation reads

$$
\rho=e^{-\beta H}
$$

and should be normalized to identity $(\operatorname{Tr} \rho=1)$ to acquire the desired statistical properties. The temperature dependent normalization factor

$$
Z=\operatorname{Tr} \exp [-\beta H]
$$

is of crucial importance, as it allows direct computation of thermodynamic quantities (entropy, inner and free energy, ...) and subsequent derivation of the thermodynamic properties (state equation) of the system. It is called a partition function.

It has been shown [20] that the partition function is not dependent on the explicit form of the scalar product. This implies that information on the thermodynamics of the sys- tem can be gained without explicit knowledge of the scalar product. This statistical approach may represent a contribution to the discussion on the ambiguity of the scalar product, in the sense that the thermodynamics of the system is insensitive with respect to the choice of the metric operator.

\section{Conclusion}

This paper was intended as a brief look into the realm of pseudo-hermitian, or $\mathcal{P} \mathcal{T}$-symmetric, quantum mechanics. To satisfy the need for more detailed information, we refer to the review article [18] and special issues of Czech. J. Phys. and J. Phys. A dedicated to $\mathcal{P} \mathcal{T}$-symmetric (pseudo-hermitian) quantum mechanics [7-11], and to the references in these publications.

In our presentation, we have tried to illustrate using examples how the formalism of pseudo-hermitian quantum mechanics can be used in the description of a physical system. Particularly, we have illustrated how this contributes to a consistent approach to the relativistic quantum mechanics of integer-spin systems.

Pseudo-hermitian operators can be used in a description of physical systems. However, their use requires a specific approach. One has to be careful about the spectrum of the operator - only operators with a real spectrum are physically admissible. Derivation of the physical properties of the system requires the construction of the new scalar product. The construction depends on the Hamiltonian, so that the scalar product is dynamically generated in this sense.

As we have remarked, the study of thermodynamic properties of the system described by pseudo-hermitian Hamiltonians represents a new stream in the field, and may provide an insight into the physical properties of these systems.

\section{Acknowledgment}

The author would like to thank the Universidad de Santiago de Chile for its kind hospitality. The work was partially supported project No. LC06002 and by GAČR grant Nr. 202/07/1307.

\section{References}

[1] Scholz, F. G., Geyer, H. B., Hahne, F. J.: Quasi-Hermitian Operators in Quantum Mechanics and the Variational Principle, Ann. Phys. Vol. 213 (1992), p. 74.

[2] Bender, C. M., Boettcher, S.: Real Spectra in Non-Hermitian Hamiltonians Having $\mathcal{P} \mathcal{T}$ Symmetry, Phys. Rev. Lett. Vol. 80 (1998), p. 5243.

[3] Bender, C. M., Boettcher, S., Meisinger, P.: PT-symmetric Quantum Mechanics, J. Math. Phys. Vol. 40 (1999), p. 2201.

[4] Dorey, P., Dunning, C., Tateo, R.: Spectral Equivalences, Bethe Ansatz Equations and Reality Properties in PT-symmetric Quantum Mechanics, J. Phys. A, Vol. 34 (2001), p. 5679.

[5] Mostafazadeh A.: Pseudo-Hermiticity versus PT Symmetry: The Necessary Condition for the Reality of the Spectrum of a Non-Hermitian Hamiltonian, J. Math. Phys. Vol. 43 (2002), p. 205. 
[6] Mostafazadeh A.: Pseudo-Hermiticity versus PT Symmetry II: A Complete Characterization of Non-Hermitian Hamiltonians with a Real Spectrum, J. Math. Phys. Vol. 43 (2002), p. 2814.

[7] Proc. $1^{\text {st }}$ Int. Workshop on Pseudo-Hermitian Hamiltonians in Quantum Physics (Editor: Znojil M.), Czech.J. Phys. Vol. 54 (2004), p. 1-156.

[8] Proc. $2^{\text {nd }}$ Int. Workshop on Pseudo-hermitian Hamiltonians in Quantum Physics (Editor: Znojil M.), Czech.J. Phys. Vol. 54 (2004), p. 1005-1148.

[9] Proc. $3^{\text {rd }}$ Int. Workshop on Pseudo-hermitian Hamiltonians in Quantum Physics (Editor: Znojil M.), Czech.J. Phys. Vol. 55 (2005), p. 1045-1 192.

[10] Special Issue Devoted to the Subject of Pseudo-Hermitian Hamiltonians in Quantum Physics (Editor: Znojil M.) Czech. J. Phys. Vol. 56 (2006), p. 885-1064.

[11] Special Issue Dedicated to the Physics of Non-Hermitian Operators (Editors: H. B. Geyer, D. Heiss, M. Znojil), J. Phys. A, Vol. 39 (2006), p. 9965-10262 and references therein.

[12] Znojil, M.: Conservation of Pseudo-Norm in PT Symmetric Quantum Mechanics, Rendiconti del Circ. Mat. di Palermo, Ser. II, Supp. Vol. 72 (2004), p. 211, arXiv: math-ph/0104012.

[13] Bender, C. M., Brody, D. C., Jones, H. F.: Complex Extension of Quantum Mechanics, Phys. Rev. Lett. Vol. 89 (2002), p. 0270401.

[14] Mostafazadeh, A.: Pseudo-Hermiticity versus PT Symmetry III: Equivalence of Pseudo-Hermiticity and the Presence of Antilinear Symmetries, J. Math. Phys. Vol. 43 (2002), p. 3944.

[15] Mostafazadeh, A.: Probability Interpretation for Klein-Gordon Fields and the Hilbert Space Problem in Quantum Cosmology, Class. Quant. Grav. Vol. 20 (2003), p. 155 .
[16] Jakubský, V., Smejkal, J.: A Positive-Definite Scalar Product for Free Proca Particle, Czech. J. Phys. Vol. 56 (2006), p. 985.

[17] Figureira de Morisson Faria, C., Fring A.: Time Evolution of Non-Hermitian Hamiltonian Systems, J. Phys. A, Vol. 39 (2006), p. 9269.

[18] Bender, C. M.: Making Sense of Non-Hermitian Hamiltonians, to be published in Rep. Prog. Phys, arXiv:hep-th/0703096.

[19] Znojil, M., Geyer, H.: Construction of a Unique Metric in Quasi-Hermitian Quantum Mechanics: Non-Existence of the Charge Operator in a $2 \times 2$ Matrix Model, Phys.Lett. B, Vol. 640 (2006), p. 52.

[20] Jakubský, V.: Thermodynamics of Pseudo-Hermitian Systems in Equilibrium, to appear in Mod. Phys. Lett. A, arXiv: quant-ph/0703092v3.

[21] Bender, C. M., Brody, D. C., Chen Jun-Hua, Jones, H. F., Milton, K. A., Ogilvie M. C.: Phys. Rev. D, Vol. 74 (2006) 025016.

[22] Case, K. M.: Some Generalizations of the Foldy-Wouthuysen Transformation, Phys. Rev. Vol. 95 (1954), p. 1323 .

Ing. Vít Jakubský, Ph.D.

phone: +420266173255

email: jakub@ujf.cas.cz

Departamento de Física

Universidad de Santiago de Chile

Casilla 307, Santiago 2, Chile

(and

phone: +420266173255

Academy of Science of the Czech Republic 25068 Rež, Czech Republic) 